\title{
A Nonlinear Map for the Decay to Equilibrium of Ideal Gases
}

\author{
Ricardo López-Ruiz \\ DIIS-BIFI, Faculty of Science \\ University of Zaragoza \\ E-50009 Zaragoza, Spain \\ Email: rilopez@unizar.es
}

\author{
Elyas Shivanian \\ Dept. of Mathematics \\ Imam Khomeini Int. University \\ Qazvin, 34149-16818, Iran \\ Email: shivanian@ikiu.ac.ir
}

\begin{abstract}
An operator that governs the discrete time evolution of the velocity distribution of an out-of-equilibrium ideal gas will be presented. This nonlinear map, which conserves the momentum and the energy of the ideal gas, has the Maxwellian Velocity Distribution (MVD) as an asymptotic equilibrium. Moreover, the system displays the increasing of the entropy during the decay to the MVD.
\end{abstract}

\section{INTRODUCTION}

A scheme inspired in economic systems that has recently been proposed [1] to explain the attractivity, and then the ubiquity, of the exponential (Boltzmann-Gibbs) Distribution (BGD) reads as follows: Let $p(m) \mathrm{d} m$ denote the PDF (probability density function) of money in a multi-agent economic system, i.e. the probability of finding an agent of the ensemble with money between $m$ and $m+\mathrm{d} m$. Consider now the discrete time evolution of an initial money distribution $p_{0}(m)$ at each time step $n$ under the action of an operator $\mathcal{T}$, which represents the average effect on the system of many random binary interactions (with number of the order of the system size) between pairs of agents exchanging their money. Thus, the system evolves from 'time $n$ to time $n+1$ to asymptotically reach the equilibrium wealth distribution $p_{f}(m)$, i.e.

$$
\lim _{n \rightarrow \infty} \mathcal{T}^{n}\left(p_{0}(m)\right) \rightarrow p_{f}(x)=B G D=\delta e^{-\delta x} \quad \text { with } \quad \delta=<p_{0}>^{-1} .
$$

In this case, $p_{f}(m)$ is the exponential distribution (BGD) with the same average wealth $\left\langle p_{f}\right\rangle=\delta^{-1}$ than the initial one $\left\langle p_{0}\right\rangle$, due to the local and total money conservation [2]. The mathematical properties of operator $\mathcal{T}$ have been disclosed in Ref. [3]. Hence, this framework not only puts in evidence that the BGD is the equilibrium distribution if not that in this case the BGD is asymptotically reached independently of the initial wealth distribution given to the system, a point of view that to date was possibly lacking in the literature.

In this work, we extend this perspective to another problem of the same statistical nature. Our goal is to explain the ubiquity of the MVD in ideal gases [4], [5]. In the next section, we explain how to obtain an operator $T$ in the space of velocity distributions in order to explain the decay of any initial velocity distribution to the MVD. Then, the dynamical properties of this operator $T$ will be sketched and some examples showing its dissipative behavior are depicted. Finally, our conclusions are given.

\section{The Model. Nonlinear Map T}

Consider an ideal gas with particles of unity mass in the three-dimensional ( $3 D)$ space. As long as there is not a privileged direction in the equilibrium, we can take any direction in the space and to study the discrete time evolution of the velocity distribution in that direction. Let us call this axis $U$. We can complete a Cartesian system with two additional orthogonal axis $V, W$. If $p_{n}(u) \mathrm{d} u$ represents the probability of finding a particle of the gas with velocity component in the direction $U$ comprised between $u$ and $u+\mathrm{d} u$ at time $n$, then the probability to have at this time $n$ a particle with a $3 D$ velocity $(u, v, w)$ will be $p_{n}(u) p_{n}(v) p_{n}(w)$. The particles of the gas collide between them, and after a number of interactions of the order of system size, a new velocity distribution is attained at time $n+1$. Concerning the interaction of particles with the bulk of the gas, we make two simplistic and realistic assumptions in order to obtain the probability of having a velocity $x$ in the direction $U$ at time $n+1$ : (1) Only those particles with an energy bigger than $x^{2}$ at time $n$ can contribute to this velocity $x$ in the direction $U$, that is, all those particles whose velocities $(u, v, w)$ verify $u^{2}+v^{2}+w^{2} \geq x^{2}$; (2) The new velocities after collisions are equally distributed in their permitted ranges, that is, particles with velocity $(u, v, w)$ can generate maximal velocities $\pm U_{\max }= \pm \sqrt{u^{2}+v^{2}+w^{2}}$, then the allowed range of velocities $\left[-U_{\max }, U_{\max }\right]$ measures $2\left|U_{\max }\right|$, and the contributing probability of these particles to the velocity $x$ will be $p_{n}(u) p_{n}(v) p_{n}(w) /\left(2\left|U_{\max }\right|\right)$. Taking all together we finally get the expression for the evolution operator $T$. This is:

$$
p_{n+1}(x)=T p_{n}(x)=\iiint_{u^{2}+v^{2}+w^{2} \geq x^{2}} \frac{p_{n}(u) p_{n}(v) p_{n}(w)}{2 \sqrt{u^{2}+v^{2}+w^{2}}} \mathrm{~d} u \mathrm{~d} v \mathrm{~d} w .
$$


Let us remark that we have not made any supposition about the type of interactions or collisions between the particles and, in some way, the equivalent of the Boltzmann hypothesis of molecular chaos would be the two simplistic assumptions we have stated on the interaction of particles with the bulk of the gas. But now a more clear and understandable framework than those usually presented in the literature appears on the scene. In fact, the operator $T$ conserves in time the energy and the null momentum of the gas. Moreover, for any initial velocity distribution, the system tends towards its equilibrium, i.e. towards the MVD. This means that

$$
\lim _{n \rightarrow \infty} T^{n}\left(p_{0}(x)\right) \rightarrow p_{f}(x)=M V D(1 D \text { case }) .
$$

In the presentation, we will pass review to the properties that (until today) have been collected here [6]. Other additional properties concerning the increasing of the entropy with time will also be given. Let us recall at this point the result that resumes the behavior of the system:

Conjecture: For any $p \in B$, with $B$ the set of functions whose norm $\|p\|$ is equal to unity, with finite mean energy, $<x^{2}, p>$, and verifying $\lim _{n \rightarrow \infty}\left\|T^{n} p(x)-\mu(x)\right\|=0$, the limit $\mu(x)$ is the fixed point $p_{\alpha}(x)=\sqrt{\frac{\alpha}{\pi}} e^{-\alpha x^{2}}$, with $\alpha=\left(2<x^{2}, p>\right)^{-1}$. In physical terms, it means that for any initial velocity distribution of the gas, it decays to the Maxwellian distribution, which is just the fixed point of the dynamics. Recalling that $\left\langle x^{2}, p\right\rangle=k \tau$, with $k$ the Boltzmann constant and $\tau$ the temperature of the gas, and introducing the mass $m$ of the particles, let us observe that the MVD is recovered in its $3 D$ format:

$$
M V D=p_{\alpha}(u) p_{\alpha}(v) p_{\alpha}(w)=\left(\frac{m \alpha}{\pi}\right)^{\frac{3}{2}} e^{-m \alpha\left(u^{2}+v^{2}+w^{2}\right)} \quad \text { with } \quad \alpha=(2 k \tau)^{-1} .
$$

Some examples showing this behavior are depicted in the Figures 1-2.

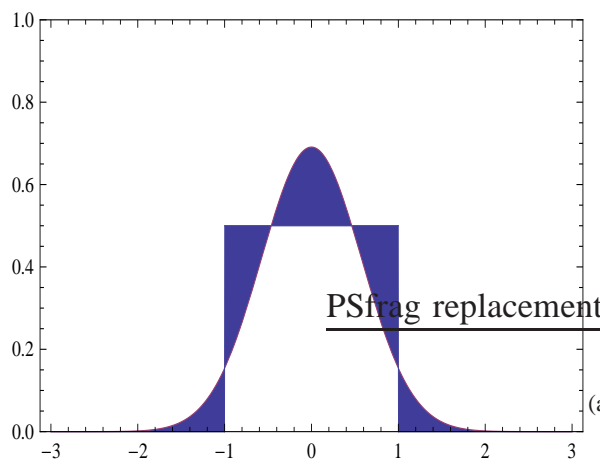

(a)

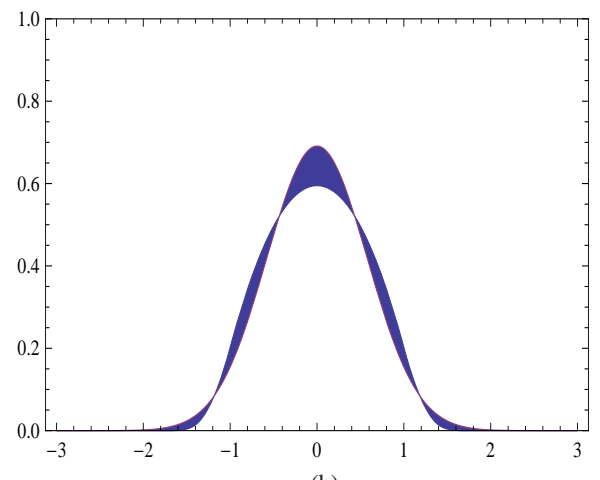

(b)

Fig. 1. (a) $p(x)$ and $\mu(x)$, (b) $T p(x)$ and $\mu(x)$.

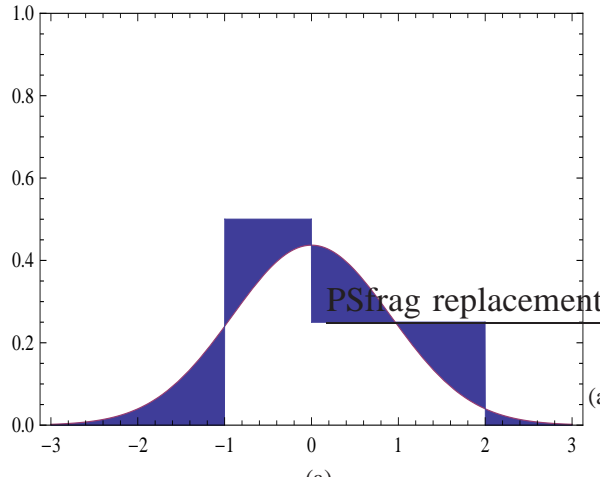

(a)

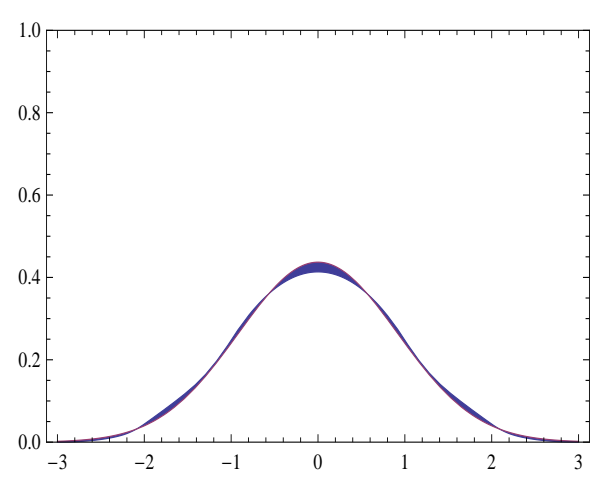

(b)

Fig. 2. (a) $p(x)$ and $\mu(x)$, (b) $T p(x)$ and $\mu(x)$.

\section{CONCLUSIONS}

In this work, a nonlinear map acting on the velocity distribution space of ideal gases, which gives account of the decay of an out-of-equilibrium velocity distribution toward the Maxwellian distribution, has been presented. Some properties and figures concerning its dynamical behavior have also been shown. 


\section{REFERENCES}

[1] R. Lopez-Ruiz, J.L. Lopez and X. Calbet, "Exponential wealth distribution: A new approach from functional iteration theory", to appear in ESAIM: Proceedings, ECIT-2010 Conference, Setember 2010; also at arXiv:1103.1501, 2011

[2] V.M. Yakovenko, Econophysics, Statistical Mechanics approach to, in Encyclopedia of Complexity and System Science, Meyers, R.A. (Ed.), Springer, Germany, 2009.

[3] J.L. Lopez, R. Lopez-Ruiz and X. Calbet, "Exponential wealth distribution in a random market. A rigorous explanation", to appear in J. Math. Anal. Appl., 2012; also at arXiv:1103.5703, 2011.

[4] L. Boltzmann, Lectures on Gas Theory, Translated by S.G. Brush, Dover Publications, New York, USA (1995).

[5] J.C. Maxwell, The scientific papers of James Clerk Maxwell, Edited by W.D. Niven, paper number XX, Dover Publications, Vol. I,II, New York, USA, 2003.

[6] E. Shivanian and R. Lopez-Ruiz, "A new model for ideal gases. Decay to the Maxwellian distribution", submitted for publication; see at arXiv:1105.4813, 2011. 